*** This paper has now been published at Behavioral and Brain Sciences, please cite the published version:

Rorot, W., Korbak, T., Litwin, P., \& Miłkowski, M. (2022). Enough blanket metaphysics, time for data-driven heuristics. Behavioral and Brain Sciences, 45, E206. doi:10.1017/S0140525X22000280***

\title{
Enough blanket metaphysics, time for data-driven heuristics
}

\author{
Wiktor Rorot ${ }^{\mathrm{a}, \mathrm{b} *}$, Tomasz Korbak ${ }^{\mathrm{c}}$, Piotr Litwin ${ }^{\mathrm{b}}$, Marcin Miłkowski ${ }_{*} *$ \\ ${ }^{a}$ University of Warsaw, Faculty of Philosophy \\ ${ }^{\mathrm{b}}$ University of Warsaw, Faculty of Psychology \\ ${ }^{c}$ University of Sussex, Department of Informatics \\ ${ }^{\mathrm{d}}$ Institute of Philosophy and Sociology, Polish Academy of Sciences \\ *w.rorot@uw.edu.pl, *mmilkows@ifispan.edu.pl
}

This paper is a commentary on Bruineberg, Dołęga, Dewhurst, and Baltieri (2021). The Emperor's New Markov Blanket. Behavioral and Brain Sciences. https://doi.org/10.1017/S0140525X21002351

\begin{abstract}
Bruineberg and colleagues criticisms' have been received but downplayed in the FEP literature. We strengthen their points, arguing that the Friston blanket discovery, even if tractable, requires a full formal description of the system of interest at the outset. Hence, blanket metaphysics is futile, and we postulate that researchers should turn back to heuristic uses of Pearl blankets.
\end{abstract}

\section{TEXT}

Bruineberg and colleagues point out an important, yet hitherto overlooked flaw in the Free Energy Principle (FEP) literature: the term "Markov blanket" has unnoticeably evolved into a more ontologically involved concept of "Friston blanket". However, the gravity of this problem has been underplayed by some of the proponents of the FEP (e.g., Wiese and Friston 2021, p. 4) who ignore the trouble that the reification of formal concepts leads to. We want to highlight one particular issue for the proponents of the FEP, especially of an associated metaphysical program of "Markovian monism" (Friston et al. 2020; Wiese and Friston 2021), concerned with the procedures for identification of Friston blankets in the world.

The problem stems from an important tension: most other fields of computational modelling use Markov blankets as approximations or optimization tools (e.g. in machine learning for the purpose of dimensionality reduction and variable selection, see Aliferis et al. 2003; Peña et al. 2007; Tsamardinos et al. 2003, or for causal search, see Bai et al. 2004; Pellet and Elisseeff 2008). However, the FEP requires an (in principle) exact identification of a unique Markov blanket for each system of interest, what Friston et al. (2021a) call a "particular partition". This is necessary since, as Friston argues (2019; Friston et al. 2020), the existence of a Markov (Friston) blanket in a (non-equilibrium) steady state system is sufficient to prove that the (autonomous, i.e. internal and active) states of the system will "look as if they are trying to minimise (...) the surprisal of states 
that constitute the thing, particle, or creature. (...) This means that anything that exists must, in some sense, be self-evidencing" (Friston et al. 2020, p. 6). Hence, for Friston, the existence of a particular partition secures that the system will conform to the FEP and allows for deducing it from first principles.

For this reason, in the recent FEP literature, there has been a quickly growing number of attempts to provide solutions to the problem of identifying Markov blankets (e.g. Friston et al. 2021a; Friston et al. 2021b; Da Costa et al. 2021). All those attempts focus on providing sufficiently strong approximations, as developing an exact analytical solution to this problem would require solving difficult open problems in partial differential equations. Additionally, researchers in this research community overlook an even more important issue, namely that both strong approximations of Markov blankets, and hypothetical methods for exact solutions to this problem require a full formal description of the system of interest (i.e., the equation describing its dynamics) at the outset. This defeats the practical purpose of finding Markov blankets.

Hence, the paradoxical tension between Markov and Friston blankets we want to highlight is that the pursuit of the metaphysical program associated with the identification of Friston blankets under the FEP entails intractable mathematical problems which depend on our prior knowledge of the system's dynamics. But if we had a formal description of the system's behavior, what new knowledge would Friston blankets provide? They certainly would not allow us to find the boundaries of entities of interest in the wild, since those must be assumed for the purpose of description of the system (even if it takes the general form of a Langevin equation, it still requires the assumption that the system is sufficiently stationary). And if we assumed the whole causal structure of the system beforehand, there would be no need to refer to Pearl nor Friston blankets to show that the system will behave in accordance with the FEP, as this would entirely follow from the description of the dynamics. As a consequence, neither this result nor blankets themselves would follow from first principles, but rather from a fallible heuristic analysis of the system of interest.

On the other hand, if we eschew precision and accept approximate optimization methods for finding Pearl blankets such as those widespread in machine learning and causal search (e.g., Pellet and Elisseeff 2008), we can use them as tools of discovery to identify the boundaries of entities (e.g., nodes in neural networks for the purpose of systems neuroscience). Furthermore, showing that a system delineated in this way conforms to the FEP might provide much more insight into the nature of the process, as it would require less knowledge at the outset. However, approximate methods do not allow for the use of the concept of Friston blanket and effectively preclude the viability of the metaphysical program of the FEP as a naturalist ontology for life sciences.

Perhaps it is too quick to throw the blankets entirely at this point. Nonetheless, we believe that the use of the Markov blanket construct should enable us to solve pressing issues in computational modelling in the sciences of brain and behavior. While Markovian monism metaphysics is not such a pressing issue, studying the causal and functional dynamics of cognitive systems is. In this context, we need various fallible heuristics for delineating Pearl blankets; that is, many stupid (Smaldino 2017), approximate and tractable models, and we need more of them to be able to make use of the error diversity inherent in any heuristic enterprise (Wimsatt 2007). While stronger analytical 
methods for finding Markov (and Friston) blankets are not necessarily dead ends, the FEP theorists' focus on those difficult methods makes them overlook a lot of lower hanging fruits.

\section{ACKNOWLEDGEMENTS}

Wiktor Rorot was supported by the Ministry of Education and Science (Poland) research Grant DI2018 010448 as part of the "Diamentowy Grant" program. Tomasz Korbak was supported by the Leverhulme Doctoral Scholarship. Marcin Miłkowski and Piotr Litwin were supported by the National Science Centre (Poland) research grant under the decision DEC2014/14/E/HS1/00803.

We want to thank Mel Andrews, Conor Heins, Dalton AR Sakthivadivel, and the Active Inference Lab for helpful clarifications.

\section{REFERENCES}

Aliferis, C. F., Tsamardinos, I., \& Statnikov, A. (2003). HITON: a novel Markov Blanket algorithm for optimal variable selection. AMLA 2003 Annual Symposium proceedings. AMLA Symposium, 2003, $21-$ 25.

Bai, X., Glymour, C., Padman, R., Ramsey, J., Spirtes, P.L., \& Wimberly, F.C. (2004). PCX : Markov Blanket Classification for Large Data Sets with Few Cases, Center for Automated Learning and Discovery, http://reports-archive.adm.cs.cmu.edu/anon/cald/CMU-CALD-04102.pdf

Da Costa, L., Friston, K., Heins, C., \& Pavliotis, G. A. (2021). Bayesian mechanics for stationary processes. Proceedings of the Royal Society A: Mathematical, Physical and Engineering Sciences, 477(2256), 20210518. https://doi.org/10.1098/rspa.2021.0518

Friston, K. J. (2019). A free energy principle for a particular physics. arXiv:1906.10184 [q-bio]. http://arxiv.org/abs/1906.10184. Accessed 29 Nov 2019

Friston, K. J., Wiese, W., \& Hobson, J. A. (2020). Sentience and the Origins of Consciousness: From Cartesian Duality to Markovian Monism. Entropy, 22(5), 516. https://doi.org/10.3390/e22050516

Friston, K. J., Fagerholm, E. D., Zarghami, T. S., Parr, T., Hipólito, I., Magrou, L., \& Razi, A. (2021). Parcels and particles: Markov blankets in the brain. Network Neuroscience, 5(1), 211-251. https://doi.org/10.1162/netn a 00175

Friston, K., Heins, C., Ueltzhöffer, K., Da Costa, L., \& Parr, T. (2021). Stochastic Chaos and Markov Blankets. Entropy, 23(9), 1220. https://doi.org/10.3390/e23091220

Pellet, J.-P., \& Elisseeff, A. (2008). Using Markov blankets for causal structure learning. Journal of Machine Learning Research, 9(43), 1295-1342.

Peña, J. M., Nilsson, R., Björkegren, J., \& Tegnér, J. (2007). Towards scalable and data efficient learning of Markov boundaries. Eighth European Conference on Symbolic and Quantitative Approaches to Reasoning with Uncertainty (ECSQARU 2005), 45(2), 211-232. https://doi.org/10.1016/i.ijar.2006.06.008

Smaldino, P. E. (2017). Models Are Stupid, and We Need More of Them. W Computational Social Psychology (s. 311-331). New York: Routledge. https://doi.org/10.4324/9781315173726-14

Tsamardinos, I., Aliferis, C. F., \& Statnikov, A. (2003). Time and sample efficient discovery of Markov blankets and direct causal relations. Proceedings of the Ninth ACM SIGKDD 
International Conference on Knowledge Discovery and Data Mining, 673-678. https://doi.org/10.1145/956750.956838

Wiese, W., \& Friston, K. J. (2021). Examining the Continuity between Life and Mind: Is There a Continuity between Autopoietic Intentionality and Representationality? Philosophies, 6(1), 18. https://doi.org/10.3390/philosophies6010018

Wimsatt, W. C. (2007). Re-engineering philosophy for limited beings: piecewise approximations to reality. Cambridge, Mass.: Harvard University Press. 